\title{
Simultaneous vs sequential discriminations of Markov-generated stimuli ${ }^{\text {? }}$
}

\author{
BILL R. BROWN AND THOMAS J. REBBIN ${ }^{2}$ \\ UNIVERSITY OF LOUISVILLE
}

This experiment required $S s$ to make same-different classifications of Markov-generated histoforms that were distortions of three different prototypes. Task memory requirements were varied by presenting stimuli either simultaneously or sequentially for comparison, and the effects of shifts in memory requirements were also assessed. Classification performance in all cases was found to be superior under the simultaneous condition. Postshift performance was strictly a function of the mode of stimulus presentation following the shift, rather than the memory requirements present in the initial trials. These results, coupled with the observed relationships between performance and a measure of individual stimulus variability under the two memory conditions, strongly suggest that prototype encoding has limited relevance to performance in the same-different classification task used here.

There have been concerted attempts by several investigators (Attneave, 1957; Evans, 1967a; Oldfield, 1954; Posner \& Keele, 1968) to formalize the loosely conceived notion that man encodes representations of commonalities among stimuli in order to effectively reduce environmental complexity. The terms schema and prototype have frequently been used to refer to such representations. The encoding of a prototype from variable stimuli and the subsequent storage of individual instances in terms of their deviations from this basic pattern, represent processes that may facilitate economy of memory and the use of retained information for recognizing complex patterns.

An extensive amount of research relevant to schema encoding has been conducted using the Markov stimuli generated by the VARGUS 7 computer system (Evans, 1967b). The VARGUS 7 computer program produces histoform stimuli that are deviations of a prototype, the latter consisting of a most probable sequence of column heights as determined by a seven-element Markov process. A population of instances, all of which are deviations from the same prototype, is termed a schema family; different schema families may be conceptualized as clusters of points in a multidimensional attribute space, and the centroid of each schema cluster constitutes the prototype for that population of instances. Constraint redundancy $\left(R_{c}\right)$, a measure discussed by Evans $(1967 \mathrm{c})$, measures the extent to which the population of instances adheres to the prototype. The $R_{c}$ variable thus reflects the tightness or looseness of each schema cluster. Within a schema cluster, the degree to which a particular instance adheres to its prototype (i.e., the cluster centroid) is reflected by the measure Proportion of Schematic Steps (POSS) developed by Bersted, Brown, \& Evans (1968). The POSS statistic varies from zero to unity; a zero POSS value would refer to a histoform that constituted a random sequence of column heights, whereas unity would refer to the actual most probable sequence of column heights designated as the prototype. As POSS increases in magnitude, a VARGUS 7 histoform of a particular schema family exhibits a progressively smaller number of deviations from the prototype for that schema cluster.

Several experiments (Bersted, Brown, \& Evans, 1969; Brown \& Dansereau, 1969; Brown \& Evans, 1969; Brown, Walker, \& Evans, 1968) have shown that Ss are able to discriminate (e.g., in a same-different classification task) among VARGUS 7 stimuli sampled from different schema families, without the benefit of knowledge of results or external reinforcement. In view of the fact that $S s$ in the above tasks were never exposed to the actual prototypes, these results have been interpreted as support for the prototype-encoding model.

The implications of the prototype-encoding concept for pattern perception rests entirely on the supposition that the process serves to reduce information processing and storage requirements (Evans, 1967a). Consequently, the following points should be considered in attempting to assess the relevance of prototype encoding to performance in categorization, oddity, and same-different classification tasks.

First, prototype encoding depends on $\mathrm{Ss}^{\prime}$ ability to identify those attributes that are distinctly representative of the different clusters of stimuli. Classification tasks that permit explicit, detailed comparisons of stimuli (e.g., simultaneous comparisons) should facilitate the acquisition of such information in comparison to tasks that do not so readily lend themselves to detailed comparisons (e.g., a sequential comparison task).

Second, given a sufficient opportunity to occur, prototype encoding should facilitate memory for pattern characteristics. Consequently, over a large number of discrimination trials, performance differences attributable to different memory requirements (i.e., simultaneous vs sequential presentation of stimuli for comparison) should diminish if prototype encoding has occurred.

Third, if the superiority of classification performance under the simultaneous presentation condition occurs as a result of the facilitation of prototype encoding (i.e., in comparison to the sequential condition), shifting Ss to a sequential task should produce a performance decrement. The level of performance following such a shift, however, would be higher than that exhibited by control Ss receiving continuous sequential trials. Similarly, shifting from a sequential to a simultaneous task should produce an increment in performance due to a reduction in memory requirements, but the level of performance following the shift would be lower than that of control Ss receiving continuous simultaneous trials. Of primary importance here is that the above predictions are based on the supposition that prototype encoding facilitates memory for pattern characteristics and occurs in both the simultaneous and sequential classification tasks, but occurs to a greater extent in the former (thus facilitating performance relative to the sequential condition).

The purpose of the experiment reported here was to evaluate the three suppositions proposed above. Specifically, the study was intended to accomplish the following objectives: (1) to compare accuracy of same-different classifications with simultaneously and sequentially presented VARGUS 7 histoforms over a large number of trials; (2) to determine the effects of shifts in memory requirements upon same-different classifications of these probabilistically generated stimuli; and 


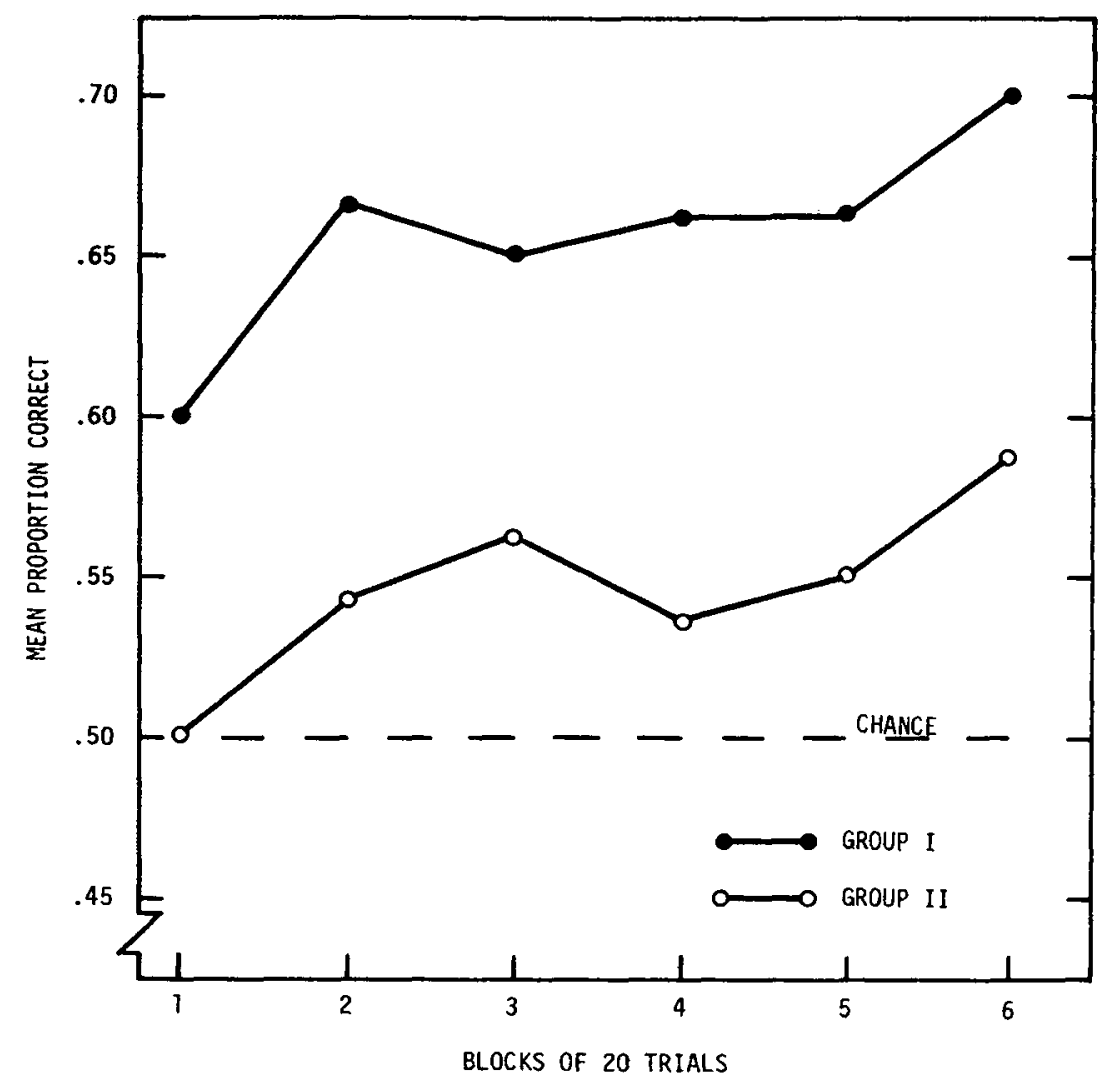

(3) to examine the relationships between same-different classifications and POSS under the simultaneous and sequential stimulus presentation conditions.

\section{METHOD}

Subjects

The Ss were 48 undergraduate students enrolled in introductory psychology courses at the University of Louisville.

\section{Stimuli}

The stimuli were 24-column histoforms generated by the VARGUS 7 computer program at $70 \% \mathbf{R}_{\mathbf{c}}$. Three distinct families of stimuli were produced by creating variants of three prototypes, which, as pointed out previously, were three different most-probable sequences of column heights. The stimulus populations from which the instances in this experiment were sampled can be found in Bersted, Brown, and Evans (1968), and are there identified as Schemata 2, 3, and 4 .

\section{Procedure}

The Ss were given an initial 60 discrimination trials with the $70 \%$ redundant VARGUS 7 stimuli by means of Xeroxed booklets. On a typical trial, 24 of the Ss viewed two stimuli on each page (simultaneous task condition) for $8 \mathrm{sec}$ and then judged them as having the same pattern or a different pattern of column heights. The remaining $24 \mathrm{Ss}$ viewed one stimulus for $4 \mathrm{sec}$, and then, after a $2-\mathrm{sec}$ interval in which they viewed a completely blank page, were instructed to turn the booklet page and study the second stimulus for $4 \mathrm{sec}$ (sequential task condition); these Ss then judged the two instances as having the same or different patterns. In both the simultaneous and sequential tasks, the Ss were not allowed to view the figures while making their responses, and they were given $4 \mathrm{sec}$ to make each same-different classification.

After the initial 60 trials, $12 \mathrm{Ss}$ (Group IS) of the 24 Ss receiving the simultaneous condition were given 60 additional trials in which the stimuli of each pair were presented sequentially. The remaining $12 \mathrm{Ss}$ (Group I) continued under the simultaneous task condition. Similarly, 12 Ss (Group IIS) of the 24 Ss receiving the sequential condition were shifted to the simultaneous task. The remaining $12 \mathrm{Ss}$ (Group II) continued under the sequential condition. The stimulus exposure and response periods following the shift were the same as those used for each condition in the initial 60 trials. The postshift stimuli were different instances from those used in the initial 60 trials, but were sampled from the same schema families.

The Ss were told in the instructions that
Fig. 1. Mean proportions of correct same-different classifications over six blocks of 20 trials for Ss receiving continous simultaneous (Group I) and sequential trials (Group II).

the two figures on any one trial did not have to be identical in order to be judged the same. Rather, the figures must merely have the same pattern, much like two different words written by the same person would have the same handwriting pattern. The Ss were told that there were three basic patterns (i.e., rather than using the term schema family) represented in the task. No knowledge of results or external reinforcement was administered at any time.

No one instance from any of the three schema families was presented more than once during the 120 trials. The two stimuli presented on each trial, whether from the same or different schema families, were paired on the basis of their having the same POSS values. Two stimulus presentation orders were obtained and these orders were used in both the simultaneous and sequential tasks. These orders were obtained in a random fashion, except for the following constraints: (1) each block of 10 trials had an equal number of same and different trials; (2) POSS was distributed identically between the blocks of 10 trials; and (3) the three schema families were approximately equally represented, in terms of both same and different pairings, within each block of 10 trials. The Ss participated in groups of two, and were assigned randomly to the four groups (and the two presentation orders within each group) as they appeared for the experimental session.

\section{RESULTS}

A two-way analysis of variance with one between-groups factor (simultaneous vs sequential presentation) and one within-groups factor (six blocks of 20 trials) was performed in order to compare performances for the two control groups (Groups I and II). The dependent variable for this analysis was the total number of correct same-different classifications. There was a significant presentation main effect, $F(1,22)=18.38, p<.001$. Figure 1 shows that mean correct classifications for the simultaneous task condition were higher across all trials blocks than for the sequential condition. No other significant effects were obtained in this analysis.

In order to compare performances for the two experimental groups (Groups IS and IIS), a two-way analysis of variance with one between-groups factor (nature of shift) and one within-groups factor (six 


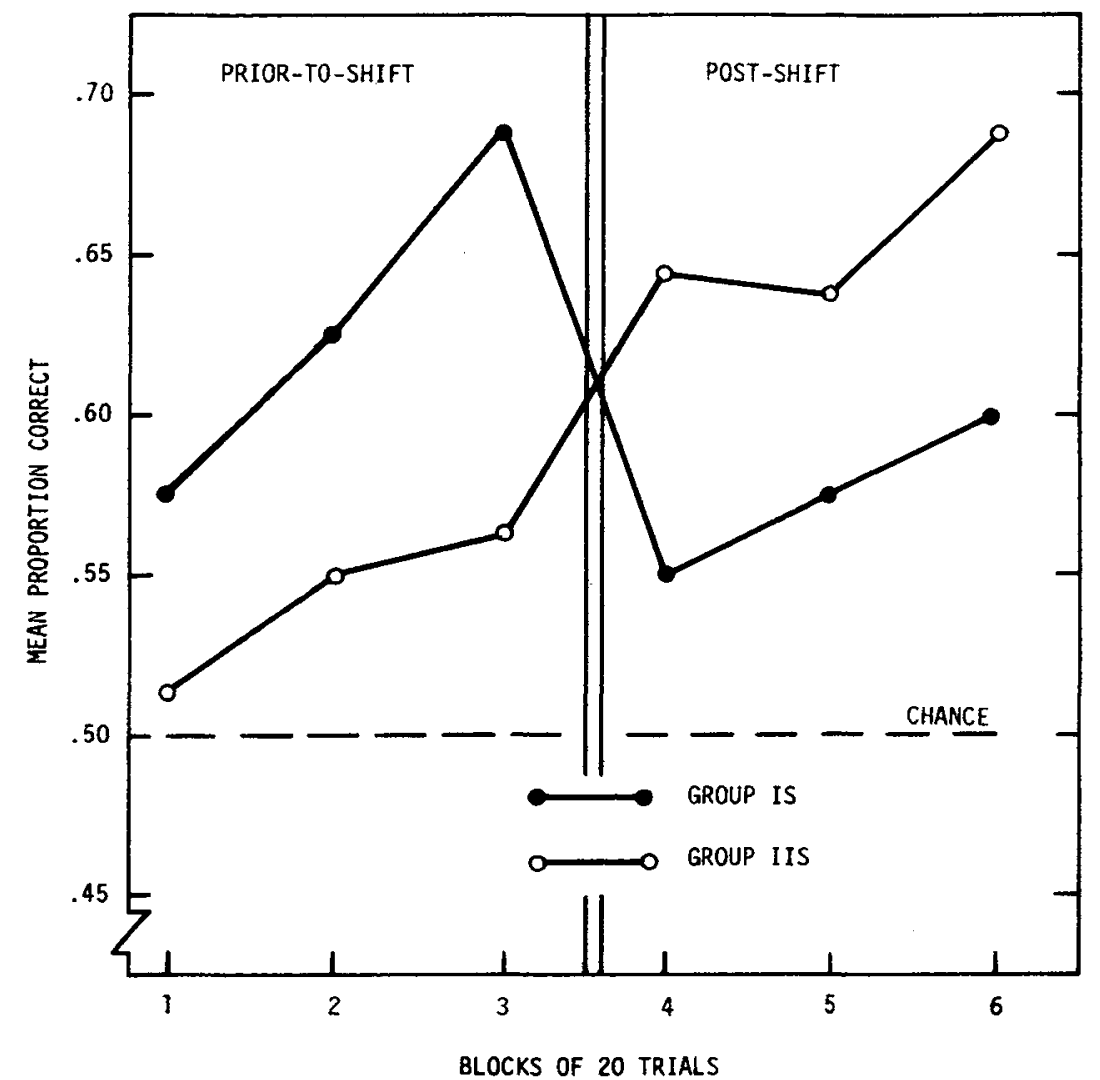

blocks of 20 trials) was performed. The dependent variable for this analysis was the total number of correct classifications. There was a significant trials main effect, $F(5,110)=2.46, p<.05$, and a significant Shift by Trials interaction, $F(5,110)=22.06, p<.01$. Figure 2 shows that during the initial 60 trials performance was higher for the simultaneous mode of presentation than for the sequential condition. Shifting to the sequential task for the former, however, produced a severe decrement in performance. Correspondingly, performance for Ss receiving the sequential condition was facilitated by the shift to the simultaneous task.

A three-way analysis of variance with two between-groups factors (prior-to-shift mode of stimulus presentation and

postshift presentation mode) and one within-groups factor (three postshift blocks of 20 trials) was performed using total correct classifications as the dependent variable. The main effect of prior-to-shift presentation mode was not significant, but the trials main effect was significant, $F(2,88)=4.01, p<.05$. There was also a significant main effect due to postshift mode of presentation, $F(1,44)=8.73$, $p<.01$. In the last 60 trials of this task, the simultaneous mode of presentation again facilitated performance in comparison to the sequential condition. Moreover, the level of performance was not significantly affected by task conditions present in the first 60 trials.

In order to determine the extent to which performance for the four groups of Ss varied as a function of POSS, one-way

Table 1

F Values Associated with POSS Main Effects $(\mathrm{df}=4 / 44)$ and Linear Trends $(\mathrm{df}=1 / 44)$ for Same and Different Trials in the Prior-To and Postshift Phases

\begin{tabular}{|c|c|c|c|c|c|c|c|c|}
\hline \multirow[b]{3}{*}{ Group } & \multicolumn{4}{|c|}{ Prior-to-Shift } & \multicolumn{4}{|c|}{ Postshift } \\
\hline & \multicolumn{2}{|c|}{ Same Trials } & \multicolumn{2}{|c|}{ Different Trials } & \multicolumn{2}{|c|}{ Same Trials } & \multicolumn{2}{|c|}{ Different Trials } \\
\hline & $F($ POSS $)$ & $F($ Linear $)$ & $\mathrm{F}(\mathrm{POSS})$ & $F($ Linear) & $F($ POSS $)$ & F(Linear) & $\mathbf{F}(\mathrm{POSS})$ & F(Linear) \\
\hline I & $5.90^{* *}$ & $15.00^{* *}$ & $3.76^{*}$ & $10.70^{* *}$ & $5.14^{* *}$ & 3.32 & $2.96^{*}$ & $9.29 * *$ \\
\hline IS & $13.26^{* *}$ & $12.04 * *$ & 2.02 & - & 1.50 & - & 1.90 & -- \\
\hline II & $3.27^{*}$ & $8.25^{* *}$ & 1.67 & $\ldots$ & $2.79^{*}$ & 3.34 & $2.98^{*}$ & $5.82^{*}$ \\
\hline IIS & 2.47 & - & $3.21^{*}$ & 1.84 & $7.97^{* *}$ & $14.47 * *$ & 1.98 & $\ldots$ \\
\hline
\end{tabular}

$* p<.05 \quad * * p<.01$
Fig. 2. Mean proportions of correct same-different classifications over six blocks of 20 trials for Ss shifted from simultaneous to sequential trials (Group IS) and from sequential to simultaneous (Group IIS).

analyses of variance with repeated measures on one factor (POSS) were computed separately for same and different trials and for the prior-to-shift and postshift trials. The dependent variable for each of these analyses was the number of correct classifications. Table 1 shows the values of the $F$ ratios associated with each of the 16 analyses and the level of significance achieved by each. In general, the POSS variable significantly influenced accuracy of classifications on both same and different trials, but performance tended to be more affected by POSS under the simultaneous than under the sequential condition.

The linear component of the sum of squares for the POSS main effect in each of the above analyses was also computed as an indication of the extent to which performance and the POSS variable were systematically related. Since the intervals between the five POSS values were not equal, a trend analysis procedure was used that is suitable for use with unequal intervals in the independent variable (see Gaito, 1965). The values of the $F$ ratios and the significance levels associated with these linear components are also presented in Table 1. It is important to note here that the significance of the linear trend was tested only for those analyses in which the main effect of the POSS factor achieved significance. The results showed that there was generally a stronger linear relation between the magnitude of POSS and performance under the simultaneous condition than under the sequential. Moreover, the linear relation was stronger for same trials in the prior-to-shift phase of the task, but was greater on different trials during the postshift phase.

Figure 3 depicts the relationship between POSS and performance for the four groups of $\mathrm{Ss}$ on same trials in the prior-to-shift and postshift phases. As the magnitude of POSS increased, accuracy of classifications also increased; this trend was stronger for the simultaneous condition than for the sequential. Figure 4 shows the relationship between POSS and performance on different trials for the four groups, and it is clear that on these trials accuracy of classifications decreased as POSS became larger. This trend was about equally represented in both prior-to-shift and postshift trials, but was stronger for 
PRIOR-TO-SHIFT

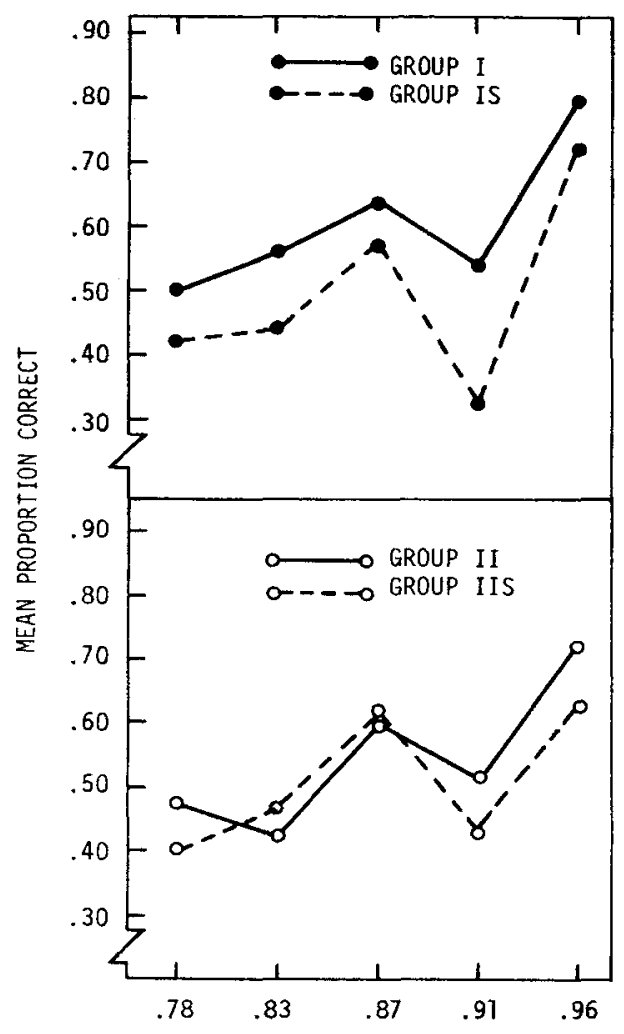

POST-SHIFT

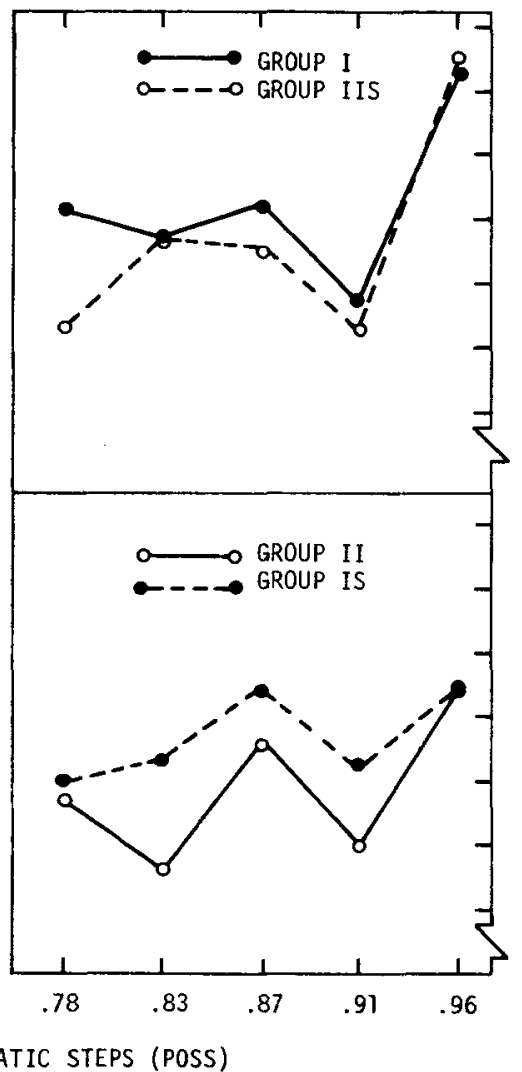

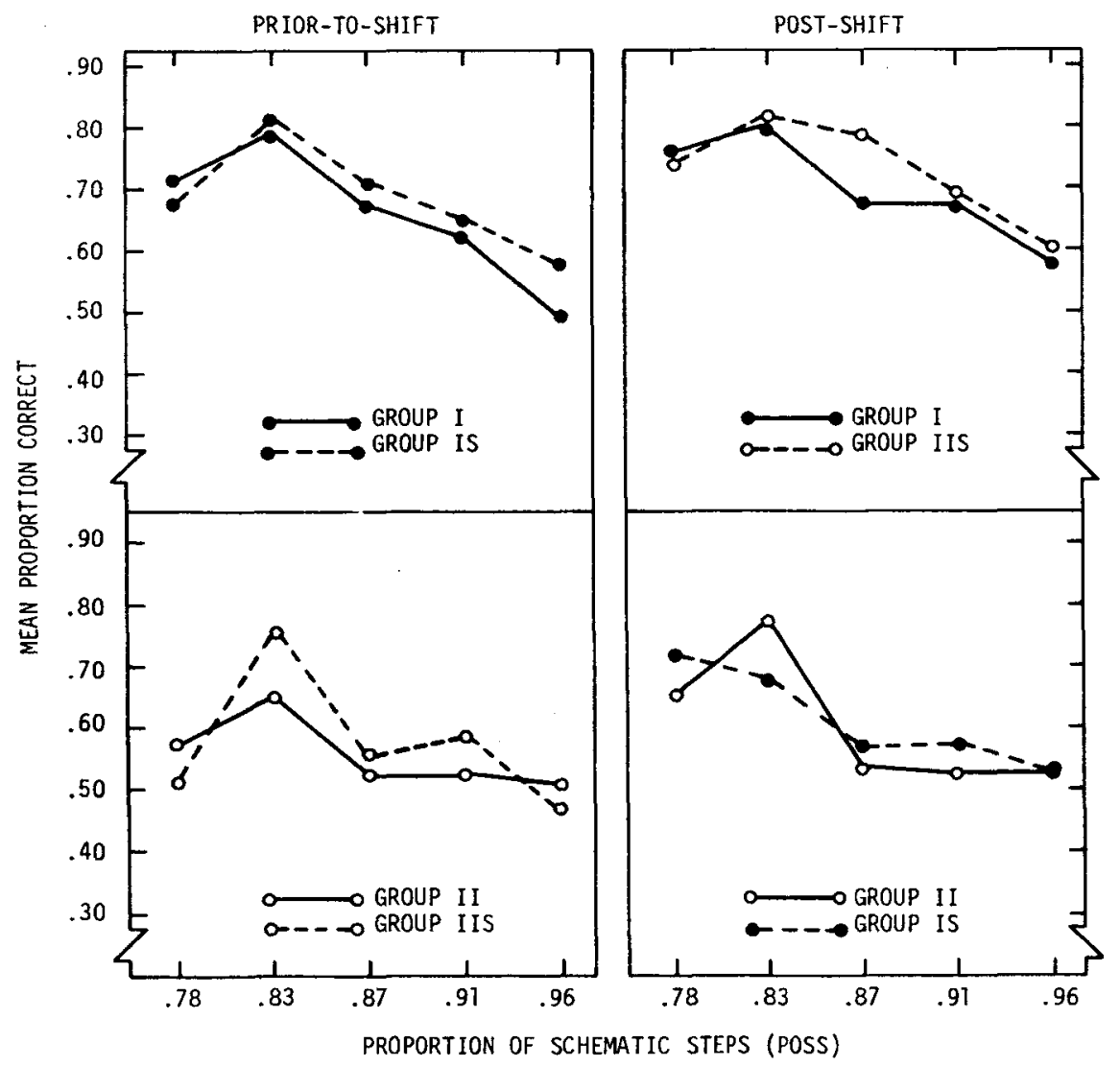

Fig. 3. Mean proportions of correct classifications on same trials (prior-to-shift and postshift) as a function of POSS. The top two quadrants represent classifications made under the simultaneous condition, whereas the lower two quadrants represent performance under the sequential condition.

the simultaneous mode of presentation than for the sequential.

\section{DISCUSSION}

The results reported here demonstrate that Ss can classify either simultaneously or sequentially presented VARGUS 7 histoforms in a manner consistent with the schema families represented in the task, and can do so without the benefit of knowledge of results or prior familiarization with the population prototypes. As expected, the simultaneous mode of presentation produced classification performance superior to that exhibited under the sequential condition. Consequently, the simultaneous task, to the extent that it permits explicit and detailed comparisons of the stimuli, evidently facilitates the detection and utilization of pattern features having some statistical association with the different schema clusters.

The hypothesis that prototype encoding, given a sufficient opportunity to occur, should reduce performance differences attributable to different memory requirements was not supported. The rates of improvement in performance for both the simultaneous and sequential tasks did not differ significantly; the simultaneous condition facilitated accuracy of discriminations early in the task and the performance curves for the two control groups did not converge even after a larger number of trials. One explanation for these findings is that the number of trials administered in this task may not have been sufficient for prototype encoding to have occurred (i.e., at least to the point where information processing and memory requirements were significantly reduced). The number of trials used here, however, was greater than that administered in the previously referenced studies that involved same-different classifications of VARGUS 7 stimuli and that claimed to

Fig. 4. Mean proportions of correct classifications on different trials (prior-to-shift and postshift) as a function of POSS. The top two quadrants represent classifications made under the simultaneous condition, whereas the lower two quadrants represent performance under the sequential condition. 
support the prototype encoding notion.

Evidence for the conclusion that prototype encoding did not occur in the present same-different task is also provided by observing the effects of shifting memory requirements. The Ss initially receiving the simultaneous condition (Group IS) exhibited a significant decrement in performance when shifted to the sequential mode of stimulus presentation. Moreover, the level of performance following the shift did not differ significantly over trials from that of Ss receiving continuous sequential trials (Group II). Similarly, Ss trained on the sequential task (Group IIS) showed a significant performance increment following a shift to the simultaneous condition, and the level of performance did not significantly differ across trials from that of $S$ s receiving continuous simultaneous trials (Group I). Since the above differences were observed over all postshift trials (i.e., rather than merely on the initial trials following the task change), accuracy of discriminations in the postshift phase of the task was primarily a function of the memory requirements of the task, regardless of whether simultaneous or sequential trials were received prior to the shift. These findings thus suggest that the performance differences associated with the simultaneous and sequential modes of stimulus presentation and which were observed in both the prior-to-shift and postshift trials cannot be attributed to different degrees of prototype encoding.

Increasing the magnitude of POSS on same trials was in general accompanied by an increasing tendency for Ss, under both the simultaneous and sequential conditions, to correctly classify instances as belonging to the same schema family. Since an increase in POSS is associated with a VARGUS 7 histoform's exhibiting a progressively smaller number of deviations from its respective prototype (i.e., the number of stimulus features statistically associated with the schema category increases), these results are consistent with the notion that $S s$ based their classifications on pattern features that were distinctly representative of the schema clusters.

Increasing the magnitude of POSS on different trials was accompanied by an increasing tendency for $S$ s to classify erroneously instances as belonging to the same schema family. Classification errors on trials involving pairings of instances from different schema families thus increased as the stimuli became closer to their respective prototypes for both the simultaneous and sequential conditions. This observation confirms the findings of two previous experiments (Bersted \& Evans, 1969; Brown \& Dansereau, 1970), but is inconsistent with the schema encoding model in that stimuli deviating only slightly from their respective prototypes (i.e., a high value of POSS) should be highly distinguishable and thus easier to classify than more variable instances with a lower POSS value.

One possible explanation for the above relationship is that $S s$ at least partially based their classifications upon the perceived "orderliness" of the stimuli. Orderliness here is defined as the number of repeated combinations of column heights that occur in the VARGUS 7 histoforms, irrespective of the particular column heights that are represented in the combinations. Regardless of the specific Markov rule used to generate the stimuli, instances having the same POSS values show exactly the same number of deviations from their respective prototypes; two stimuli on any one trial that are paired on the basis of their having the same POSS value thus would appear to be equally orderly, and would be erroneously judged as belonging to the same category even if they were sampled from different schema families. Moreover, as POSS increases in magnitude, the orderliness of the stimuli would increase. In the case of stimuli sampled from different schema categories, classification errors resulting from making judgments based on the orderliness of the stimuli would be expected to increase as a function of POSS. On the other hand, if any two stimuli were sampled from the same schema family (and were characterized by the same POSS values), a classification based on the orderliness dimension would be correct, and the number of correct classifications would be expected to increase as POSS values became larger.

In the postshift phase of the experiment, the degree to which performance was linearly related to POSS was generally higher for the simultaneous condition than for the sequential (Groups I and IIS vs Groups II and IS), regardless of whether the $S$ s received the simultaneous or sequential modes of stimulus presentation in the initial 60 trials of the task. These results also support the conclusion that accuracy of classifications in the postshift phase was primarily a function of the memory requirements of the task, rather than the mode of stimulus presentation received prior to the shift. Together, these findings indicate that prototype encoding did not occur to any significant degree in the present experiment; to the extent that Ss did encode stimulus features statistically associated with the different schema clusters, the information acquired was not sufficient to reduce performance differences attributable to different memory requirements.

The results reported here indicate that the prototype encoding model, as presently conceptualized, has limited relevance to same-different classifications of VARGUS 7 stimuli. The need for more rigorous delineation and assessment of the model's suppositions in a variety of pattern-perception tasks having different memory and processing requirements is thus clearly indicated.

\section{REFERENCES}

ATTNEAVE, F. Transfer of experience with a class-schema to identification learning of patterns and shapes. Journal of Experimental Psychology, 1957, 54, 81-88.

BERSTED, C. T., \& EVANS, S. H. Distance from the schema as a predictor of classification and similarity judgments. Paper presented at annual meeting of the Psychonomic Society, November 1969.

BERSTED, C. T., BROWN, B. R., \& EVANS, S. H. A standard set of VARGUS 7 patterns at three levels of schematic redundancy. Psychonomic Monograph Supplements, 1968, 2, 251-282.

BERSTED, C. T., BROWN, B. R. \& EVANS, S. H. Free sorting of stimuli clustered in a multidimensional attribute space. Perception \& Psychophysics, 1969, 6, 409-413.

BROWN, B. R., \& DANSEREAU, D. F. Discrimination among schematic stimuli as a function of response mode, constraint redundancy, and form of Markov rule. Psychonomic Science, 1969, 17, 197-198.

BROWN, B, R \& DANSEREAU, D. $F$ Functional equivalence between same-different classifications and judged similarity of Markov patterns. Perception \& Psychophysics, 1970, 7 , 307-310.

BROWN, B. R., \& EVANS, S. H. Perceptual learning in pattern discrimination tasks with two and three schema categories. Psychonomic Science, 1969, 15, 101-103.

BROWN, B. R., WALKER, D. W., \& EVANS, S. H. Schematic concept formation as a function of constraint redundancy and knowledge of results. Psychonomic Science, 1968, 11, 75-76.

EVANS, S. H. A brief statement of schema theory, Psychonomic Science, 1967a, 8, 87-88.

EVANS, S. H. VARGUS 7: Computer pattern from Markov processes. Behavioral Science, $1967 b, 12,323.328$.

EVANS, S. H. Redundancy as a variable in pattern perception. Psychological Bulletin, $1967 \mathrm{c}, 67,104-113$.

GAITO, J. Unequal intervals and unequal $n$ in trend analyses. Psychological Bulletin, 1965, 63, 125-127.

OLDFIELD, R. C. Memory mechanisms and the theory of schemata. British Journal of Psychology, 1954, 45, 14-23.

POSNER, M. I., \& KEELE, S. W. On the genesis of abstract ideas. Journal of Experimental Psychology, 1968, 77, 353-363.

\section{NOTES}

1. This research was conducted by the Performance Research Laboratory and supported by a Department of Defense Project THEMIS contract, No. HC19-69-C-0009, under the Department of the Army.

2. Address: Department of Psychology, University of Louisville, Louisville, Kentucky 40208 .

(Accepted for publication February 2, 1970.) 